8th Alexander Friedmann International Seminar

on Gravitation and Cosmology

International Journal of Modern Physics: Conference Series

Vol. 3 (2011) 195-202

(C) World Scientific Publishing Company

DOI: $10.1142 / \mathrm{S} 2010194511001280$

\title{
THE POINCARÉ CONJECTURE AND THE COSMOLOGICAL CONSTANT
}

\author{
M. D. MAIA \\ Instituto de Física, Universidade de Brasília, Brasília Distrito Federal. 70910-900 Brazil \\ maia@unb.br
}

Received 11 June 2011

Revised 1 July 2011

\begin{abstract}
The concept of deformation of Riemannian geometry is reviewed, with applications to gravitation and cosmology. Starting with an analysis of the cosmological constant problem, it is shown that space-times are deformable in the sense of local change of shape. These deformations leave an observable signature in the space-time, characterized by a conserved tensor, associated with a tangent acceleration, defined by the extrinsic curvature of the space-time. In the applications to cosmology, we find that the accelerated expansion of the universe is the observable effect of the deformation, dispensing with the cosmological constant and its problems.
\end{abstract}

Keywords: Manifold deformations; Poincaré conjecture; cosmological constant.

PACS numbers: 98.80.-k, 98.80.Es, 95.30.S, 95.36.+X

\section{The Cosmological Constant Problem}

The cosmological constant $\Lambda$ was introduced and removed by Einstein in 1917, basically because its presence is not compatible with the Minkowski space-time as a solution of his gravitational equations. Yet, the cosmological constant remains as the basic explanation for the observed acceleration of the universe, within the so called $\Lambda$ CDM paradigm.

The usual physical interpretation of $\Lambda$ is that of the vacuum energy density $<\rho_{v}>$ of quantum fields. This follows from the semiclassical equations with $\Lambda:^{1}$

$$
R_{\mu \nu}-\frac{1}{2} R g_{\mu \nu}-\Lambda g_{\mu \nu}=-8 \pi G<\rho_{v}>g_{\mu \nu} .
$$

These equations holds true only under the condition that $\Lambda g_{\mu \nu}$ in the left hand side cancels with the $8 \pi G\left\langle\rho_{v}>g_{\mu \nu}\right.$ in the right hand side. In this case we obtain an equation compatible with the Minkowski tangent solution, with the Poincaré symmetry and the usual quantum field theory (QFT) required for the evaluation of the vacuum energy.

As it happens, the theoretical estimates give the value

$$
<\rho_{v}>\approx 10^{76} \mathrm{GeV}^{2} / \mathrm{c}^{4} \text {. }
$$


On the other hand, the acceleration of the universe measured by the CMBR in various precision experiments, indicates that

$$
\Lambda / 8 \pi G \approx 10^{-47} \mathrm{GeV}^{2} / \mathrm{c}^{4}
$$

and there is no known procedure in QFT capable of adjusting these values. ${ }^{2,3}$

Since the cosmological constant term is the only allowed addition to Einstein's tensor, following the contracted Bianchi identity, regardless of the existence and the nature of the energy-momentum tensor, we conclude that the cosmological constant term in Einstein's equations is a quantity of geometric nature which is independent of the right hand side of Einstein's equations.

That conclusion implies that the difference between $\Lambda$ and $\left\langle\rho_{v}\right\rangle$ is not only numerical, but mainly conceptual: The presence of $\Lambda$ does not depend on the nature of what is placed in the right hand side of Einstein's equations. Such conceptual difference is a consequence of the topological difference between the Minkowski and the de Sitter space-times with their respective groups of isometries. As we know, it is possible to make a group contraction from the latter to the former, but at the cost of the limit $\Lambda \rightarrow 0$. However, this limit is not followed by the continuous change of shape of the respective space-times. As a sphere cannot be be continuously transformed into a plane without stretching and tearing off the manifold, the de Sitter group contraction is not followed by a smooth deformation of the respective geometries. The recent solution of the Poincaré conjecture on the deformation of Riemannian manifolds and its generalization provided by Nash's theorem, offers a possible to topological solution of the cosmological constant problem.

\section{The Poincaré Conjecture}

In 1904 Henry Poincaré conjectured that a 3-dimensional (compact and simply connected) manifold can be continuously deformed into a 3-sphere. Although it is a very intuitive problem it was formally proved only recently in 2006 by Grigori Perelman, using the Ricci flow equation derived by Richard Hamilton in $1982,{ }^{4-7}$ given by

$$
R_{\mu \nu}=-\frac{1}{2} \frac{\partial g_{\mu \nu}}{\partial y}
$$

One simple way to derive this equation is to write the Ricci tensor as

$$
R_{\mu \nu}=(\log \sqrt{g})_{, \mu \nu}-\Gamma_{\mu \nu, \rho}^{\rho}+\Gamma_{\mu \rho}^{\sigma} \Gamma_{\nu \sigma}^{\rho}-\Gamma_{\mu \nu}^{\rho}(\log \sqrt{g})_{, \rho} .
$$

From which, using geodesic coordinates, we obtain the the Ricci scalar

$$
R=g^{\mu \nu} R_{\mu \nu}=\nabla^{2}(\log \sqrt{g}) .
$$

On the other hand, replacing $u=\log \sqrt{g}$ in the Fourier heat equation $\nabla^{2} u=\frac{\partial u}{\partial t}$ we find that

$$
\nabla^{2}(\log \sqrt{g})=g^{\mu \nu} \frac{1}{2} \frac{\partial g_{\mu \nu}}{\partial t}
$$


Comparing the right hand side of (2) with the left hand side of (3), removing the trace and replacing $t$ by an arbitrary coordinate $y$, we obtain the Ricci flow condition (1), up to a sign and to an arbitrary anti-symmetric tensor.

As it happens with Einstein's equations, the Ricci flow is not native to Riemannian geometry, but it represents an additional postulate describing the variation of the metric along a given direction, not necessarily time-like.

The merit of the Hamilton/Perelman's results lies in the interpretation of Fourier's heat flow in terms of the continuous deformation of the geometry which can be exemplified as follows: Consider the example of a closed surface $S$ being orthogonally crossed by a number of flow lines per unit of area, originating from the heat flux in a heating body. Then, draw another surface, for example a spherical surface, $S_{0}$ inside $S$, such that the number of flux lines per unit of area orthogonally crossing it, is the same as that for $S$. Next, freeze these flux lines, and use them as guides to smoothly deform $S$, always keeping it orthogonal to the flux lines, without breaking the surface or even without making wrinkles, until reaching $S_{0}$. Under such conditions we say that the surface $S$ has been smoothly deformed into $S_{0}$, in accordance with (1).

It is a simple matter to see that the Ricci flow is not compatible with general relativity: Writing Einstein's equations as

$$
R_{\mu \nu}=8 \pi G\left(T_{\mu \nu}-\frac{1}{2} T g_{\mu \nu}\right)
$$

and comparing with (1), we obtain

$$
\frac{\partial g_{\mu \nu}}{\partial y}=-16 \pi G\left(T_{\mu \nu}-\frac{1}{2} T g_{\mu \nu}\right)
$$

with the inevitable conclusion that the Ricci flow forces the Einstein gravitation to propagate linearly on any direction $y$ of space-time, which does not make sense in general relativity, even considering only its linear approximation. Either we use the hyperbolic Einstein's equations or the Parabolic Ricci flow equation (1). The so called Ricci-flow cosmology would be an entirely new proposition. ${ }^{8}$

In the following we briefly review a more general and older concept of deformation in Riemannian geometry which is compatible with general relativity and with the present cosmology, where instead of the surface $S$ we have a solution of Einstein's equations.

\section{The Nash Geometric Flow}

Perelman's demonstration of the Poincaré conjecture suggests the necessity of a mechanism capable of modifying the shape of a manifold, that could be universally applied to all Riemannian geometries, without imposing a constraint to the metric, independently of its dimension and metric signature. Such general deformation 
process was derived by John Nash in 1956 and it is given by

$$
k_{\mu \nu}=-\frac{1}{2} \frac{\partial g_{\mu \nu}}{\partial y}
$$

where $k_{\mu \nu}$ denotes the extrinsic curvature of the embedded Riemannian geometry.

The expression (4) was proposed by James York, restricted to 3-dimensional space-like surfaces evolving in a space-time, as a way to establish the initial value conditions in the ADM formulation of general relativity. As we know, the ADM proposition did not work for its intended purposes, namely to define a canonical formulation of general relativity and its eventual use to quantize gravitation, because its incompatibility with the diffeomorphism invariance of general relativity, which leads to a constrained Hamiltonian. Even using Dirac's procedure for constrained systems it did not work because the Poisson bracket structure is not covariant with the group of diffeomorphisms.

The diffeomorphism invariance of general relativity is a statement about which observers are allowed in the theory, namely all. As such, the diffeomorphism invariance has to do with the observable-observer relation and therefore it is a fourdimensional characteristic. This means that in an embedded space-time the diffeomorphism invariance must remain confined to the four-dimensional space-time where the observers and their gauge fields are defined. In other words, there is no physical reason to assume that it would extend to the extra dimensions. Hence, in an embedded space-time the diffeomorphism invariance holds in the four-dimensional subspace only.

For some authors, the embedding of the space-time into another manifold is considered to be just as a mathematical property, without physical significance. ${ }^{9}$ This is not true as we shall see. Indeed the embedding of Riemannian manifolds has its origins in the solution of local shape problem. That is, in the inability of the Riemann curvature tensor to specify the local shape of the manifold. ${ }^{10}$ In the following, we will show that an isometric embedding of the space-time produces an observable effect. In particular for a space-time it can be detected as a physical observable.

The original derivation of (4), given in part A of Nash's paper was unduly complicated by the use of smoothing operators. ${ }^{11}$ To show it in a simpler form, we may start with the case of only one extra dimension. The case of many extra dimensions was considered in Ref. 12 leading to the same conclusion.

The concepts of flux and flow lines introduced by Fourier can be formally defined as the trajectories of a one-parameter groups of diffeomorphisms on a Riemannian sub manifold $S$, embedded into another $V_{D}$, defined as follows: Given a point $p \in$ $S$ and the unit normal vector $\eta$ at $p$, we obtain a curve (called the orbit of $p$ ) with parameter $y, \alpha(y)=h_{y}: S \rightarrow V_{D}$, orthogonal to $S$, with velocity vector $\alpha^{\prime}(y)=\eta$. The diffeomorphism property means that these orbits can be generated by infinitesimal increments of the parameter $y$, so that subsequent points are defined by a composition law given by $h_{y}(p) o h_{y}^{\prime}(p)=h_{y+y^{\prime}}(p)$. The group property follows 
from the definitions $h^{-1}(p)=h_{-y}(p)$ and $h_{0}(p)=p$. Given a geometrical object $\Omega$ defined in $S$, it propagates along these orbits by the Lie transport ${ }^{13}$

$$
\Omega^{\prime}=\Omega+y £_{\eta} \Omega .
$$

\section{Embedded Space-Times}

Denoting the metric of the five-dimensional embedding space $V_{5}$ by $\mathcal{G}_{A B}, A, B=$ $1, \ldots, 5$, the isometric embedding of a Riemannian geometry $S$ a map $X: S \rightarrow V_{5}$, such that

$$
X_{, \mu}^{A} X_{, \nu}^{B} \mathcal{G}_{A B}=g_{\mu \nu}, X^{A}{ }_{, \mu} \eta^{B} \mathcal{G}_{A B}=0, \eta^{A} \eta^{B} \mathcal{G}_{A B}=1 .
$$

The four-dimensionality of space-time is a consequence of the dual properties of gauge fields, which consists of our main observational tools. By definition, the embedding of a space-time maintains that dimensionality, but it adds a topological information by the extrinsic curvature. This is a measure of the variation of the normal vector $\eta$ when its foot is displaced in a tangent direction to $S$, projected into $S$ :

$$
\eta_{, \mu}^{A}=-k_{\mu}^{\rho} X_{, \rho}^{A}
$$

To understand the meaning of this extrinsic curvature, we may picture the orbits of points in $S$ with tangent vector $\eta$ as plane curves in $V_{5}$, whose acceleration $\eta^{\prime}$ is orthogonal to $\eta$ (following the Frenet equation). Therefore, it follows from (5) that $\eta^{\prime}$ is a vector tangent to $S$. From the above expression it follows that the extrinsic curvature represents an acceleration in $S$, giving a mechanical meaning to Nash's deformation.

The vectors $\left\{X_{, \mu}\right\}$ define a tangent basis to $S$, so that $\left\{X_{\mu}^{A}, \eta_{a}^{A}\right\}$ define a Gaussian frame of $V_{5}$. To obtain (4) consider the Lie transport of that Gaussian frame along the orbits, giving a new set of vector fields

$$
X^{\prime A}=X^{A}+y £_{\bar{\eta}} X^{A}=X_{, \mu}^{A}+y \quad \eta_{, \mu}^{A}, \quad \eta^{\prime A}=\eta^{A}+y £_{\eta} \eta=\eta^{A} .
$$

Nash's local embedding theorem consists in showing that $X^{\prime A}$ define a new deformed manifold $S^{\prime}$ provided they satisfy similar isometric embedding equations

$$
X_{, \mu}^{\prime A} X^{\prime B}{ }_{, \nu} \mathcal{G}_{A B}=g_{\mu \nu}^{\prime}, \quad X^{A}{ }_{, \mu} \eta^{B} \mathcal{G}_{A B}=0, \quad \eta^{A} \eta^{B} \mathcal{G}_{A B}=1,
$$

where $g_{\mu \nu}^{\prime}$ is the deformed metric. Replacing $X^{\prime A}{ }_{, \mu}$ given by (7) in these equations we obtain in a straightforward way that

$$
\begin{aligned}
& g_{\mu \nu}^{\prime}=g_{\mu \nu}-2 y k_{\mu \nu}+y^{2} g^{\rho \sigma} k_{\mu \rho} k_{\nu \sigma} \\
& k_{\mu \nu}^{\prime}=k_{\mu \nu}-2 y g^{\rho \sigma} k_{\mu \rho} k_{\nu \sigma} .
\end{aligned}
$$

Deriving the first equation with respect to $y$, and comparing with the second equation we obtain Nash's geometric flow (4). Notice that in each deformation obtained 
by (4), the extrinsic curvature is independent of the metric, satisfying the GaussCodazzi equations for the embedded manifold. Thus, for any particular deformation the variable $y$ is contained in the expression of the extrinsic curvature.

The isometric condition (5) means that the geometry of the embedded space is induced by the geometry of the embedding space (so that we have only one metric geometry). Therefore, to obtain the same gravitational field definition, the metric of the embedding space must be defined by the same Einstein-Hilbert variational principle, leading to the the higher dimensional Einstein's equations (in arbitrary coordinates)

$$
{ }^{5} \mathcal{R}_{A B}-\frac{1}{2}{ }^{5} \mathcal{R} \mathcal{G}_{A B}=G_{*} T_{A B}, \quad A, B=1, \ldots, 5,
$$

where $G_{*}$ is the appropriate gravitational constant (including the solid angle) for a 5-dimensional space. ${ }^{14}$

To obtain the four-dimensional equations we only have to write the above equations in the Gaussian frame $\left\{X_{, \mu}^{A}, \eta^{A}\right\}$ of the embedding space. ${ }^{\text {a }}$ We obtain two sets of equations:

$$
\begin{aligned}
& R_{\mu \nu}-\frac{1}{2} R g_{\mu \nu}-Q_{\mu \nu}=-8 \pi G T_{\mu \nu}, \\
& k_{\mu ; \rho}^{\rho}-h_{, \mu}=0, \quad \mu, \nu=1, \ldots, 4,
\end{aligned}
$$

where we have denoted the tensor quantity

$$
Q_{\mu \nu}=k_{\mu}^{\rho} k_{\rho \nu}-h k_{\mu \nu}-\frac{1}{2}\left(K^{2}-h^{2}\right) g_{\mu \nu}
$$

It follows directly from this definition that

$$
Q_{; \nu}^{\mu \nu}=0
$$

so that in principle $Q_{\mu \nu}$ corresponds to an observable in the space-time, in the sense of Noether's theorem.

It should also be noted that the definition of the embedding geometry by the higher dimensional equations (8) provides a more general embedding than the common practice of specifying a particular 5-dimensional embedding space, such as a flat space, or a de Sitter/anti-deSitter spaces, or a Ricci-flat embedding space. Since the metric of the embedded space-time is induced by that of the embedding space, these particular choices, imply in a constrained embedding. For example, when the 5-dimensional embedding space is flat, the Gauss-Codazzi embedding equations do not have a solution for specific metrics. ${ }^{15,16}$ In the case of Ricci-flat 5-dimensional embedding space, we obtain from (8) that the Ricci tensor of the space-time is not zero but it must be conserved. ${ }^{17,18}$ Finally in the Randall-Sundrum braneworld models the extrinsic curvature is not dynamical, but rather given by the four-dimensional confined sources.

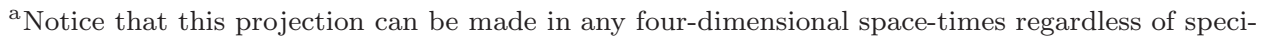
fying the value of $y$. 
On the other hand, in the geometric deformation of Nash with the EinsteinHilbert principle, the four-dimensional gravitational equations is defined by the same four-dimensional Einstein's tensor plus the additional tensor $Q_{\mu \nu}$ in place of the cosmological constant.

Since $Q_{\mu \nu}$ is conserved, it must correspond to an observable quantity. Indeed, the standard Friedmann-Lemaitre-Robertson-Walker (FLRW) universe is described by the classical Friedman's equation

$$
\dot{a}^{2}+k \approx-\frac{8 \pi G}{3} \rho a^{2}
$$

However, for the FLRW universe embedded in a five-dimensional space defined by (8), Friedman's equations becomes

$$
\dot{a}^{2}+k \approx-\frac{8 \pi G}{3} \rho a^{2}+\frac{b^{2}}{a^{2}}
$$

where we have denoted $a(t)=g_{11}$ and $b(t)=k_{11}$. The term $b^{2} / a^{2}$ corresponds to the deformation tensor $Q_{\mu \nu}$.

In a previous publication we have compared the effect of $b^{2} / a^{2}$ with the effect of a hypothetic phenomenological fluid, the x-fluid idealized by Turner and White to simulate the gravitational effect of dark energy. ${ }^{19}$ In a subsequent publication we have generalized that result in two ways: First, instead of a phenomenological fluid the extrinsic curvature was regarded as an independent spin-2 field, second, by using a model independent statistical analysis based on the luminosity-distance measurements. In both cases we have found that the deformation term $b^{2} / a^{2}$ is sufficient to describe the acceleration of the universe. More importantly, we have found that the cosmological constant does not play any significant role on this deformation. $^{20}$

One interesting question concerns the end of the acceleration. When the extrinsic curvature is proportional to the metric we find a peculiar situation: Admitting that $k_{\mu \nu}=\alpha_{0} g_{\mu \nu}$, the space-time contains only umbilic points and it behave as a constant curvature space. In such cases the Nash deformation ceases and (11) gives

$$
Q_{\mu \nu}=3 \alpha_{0}^{2} g_{\mu \nu}
$$

Replacing these conditions in (10) it becomes an identity while in (9) we obtain Einstein's equations with a cosmological constant term, representing the end limit of the deformation process.

\section{Conclusions}

We conclude that the inclusion of a cosmological constant has led us to a sequence of conceptual and observational difficulties. In particular its comparison with the vacuum energy density in quantum field theory cannot be sustained, mainly because the cosmological constant would exist independently of the existence and nature of the energy-momentum tensor. By comparing such problem with the Poincaré conjecture we conclude that the problem could be solved if we include in Einstein's 
gravitational theory a smooth deformation mechanism capable of modifying spacetime topology. Such mechanism exists and it is given by the geometric deformation introduced by Nash in 1956, based on the foundations of Riemannian geometry. It is entirely compatible with general relativity, but it adds a new observable quantity constructed with the extrinsic curvature of the space-time. When this is implemented in the FLRW cosmology, we find that this conserved quantity corresponds to the observed acceleration of the universe. We also find that such acceleration ends in a constant curvature de Sitter phase.

\section{References}

1. Ya. B. Zel'dovich and I. D. Novikov, The Structure and Evolution of the Universe (Chicago University Press, Chicago, 1983).

2. S. Weinberg, Rev. Mod. Phys. 61, 1 (1989).

3. S. Weinberg, The cosmological constant problems, arXiv:astro-ph/0005265.

4. R. Hamilton, J. Differential Geom. 17, 255 (1982).

5. G. Perelman, The entropy formula for the Ricci flow and its geometric applications, arXiv:math.DG/0211159.

6. G. Perelman, Ricci flow with surgery on three-manifolds, arXiv:math.DG/0303109.

7. G. Perelman, Finite extinction time for the solutions to the Ricci flow on certain three-manifolds, arXiv:math.DG/0307245.

8. T. Buchert and M. Carfora, Class. Quant. Grav. 19, 6109 (2002).

9. C. W. Misner, K. S. Thorne and J. A. Wheeler, Gravitation (W. H. Freeman Co., New York, 1970).

10. L. Schlaefli, Annali di Matematica (2 ${ }^{\text {nd }}$ ser.) 5, 170 (1871).

11. J. Nash, Ann. Math. 63, 20 (1956).

12. M. D. Maia, N. Silva and M. C. B. Fernandes, JHEP 0704, 047 (2007).

13. M. Crampin and F. A. E. Pirani, Applicable Differential Geometry (Cambridge University Press, Cambridge, 1986).

14. N. Arkani-Hamed et al., Phys. Lett. B 429, 263 (1998).

15. E. Kasner, Am. J. Math. 48, 126 (1921).

16. P. Szekeres, Nuovo Cimento 43, 1062 (1966).

17. J. E. Campbell, A Course of Differential Geometry (Claredon Press, Oxford, 1926).

18. F. Dahia and C. Romero, Braz. J. Phys. 35, 1140 (2005).

19. E. M. Monte, J. M. F. Maia and J. S. Alcaniz, Class. Quant. Grav. 22, 1623 (2005).

20. M. D. Maia, J. S. Alcaniz, E. M. Monte and A. J. S. Capistrano, The deformable Universe, arXiv:1101.3951; to appear in Gen. Rel. Grav. (2011). 